\title{
All-optical Modulation in Germanium-on-silicon Waveguides in the Mid-infrared
}

\author{
L. Shen, N. Healy, C. J. Mitchell, J. Soler. Penades, M. Nedeljkovic, G. Z. Mashanovich, \\ and A. C. Peacock* \\ Optoelectronics Research Centre, University of Southampton, Southampton SO17 1BJ, United Kingdom \\ *acp@orc.soton.ac.uk
}

\begin{abstract}
All-optical modulation is demonstrated in low loss germanium-on-silicon waveguides at mid-infrared wavelengths. The results indicate the suitability of this platform for optical signal processing in this long wavelength regime.
\end{abstract}

(C) 2014 Optical Society of America

OCIS codes: $160.6000,230.7370,230.4110$

\section{Introduction}

Group IV mid-infrared (mid-IR) photonics is currently attracting considerable attention for applications in sensing and spectroscopy as this wavelength region contains strong vibrational signatures for a number of gases and molecules. Although much of the work in this area has leveraged the well-developed silicon-on-insulator (SOI) platform, more recently several demonstrations of low loss germanium-on-silicon (Ge-on-Si) waveguides have emerged [1,2]. Here we report the first demonstration of all-optical modulation in a Ge-on-Si rib waveguide using two different absorption mechanisms. The first is a free carrier absorption (FCA) scheme, where it is possible to employ a standard telecoms source as the pump, and the second makes use of the strong two-photon absorption (TPA) effect in the $2 \mu \mathrm{m}$ region for cross amplitude modulation (XAM). Modulation is demonstrated for selected wavelengths across the $2-3.2 \mu \mathrm{m}$ region, obtaining extinction ratios as high as $\sim 8 \mathrm{~dB}$, at speeds of tens of $\mathrm{MHz}$ to hundreds of $\mathrm{GHz}$. The low losses that have been measured in these waveguides out to $3.8 \mu \mathrm{m}$ suggest it would be straightforward to extend the modulation further into the mid-IR.

\section{Germanium-on-silicon waveguides}
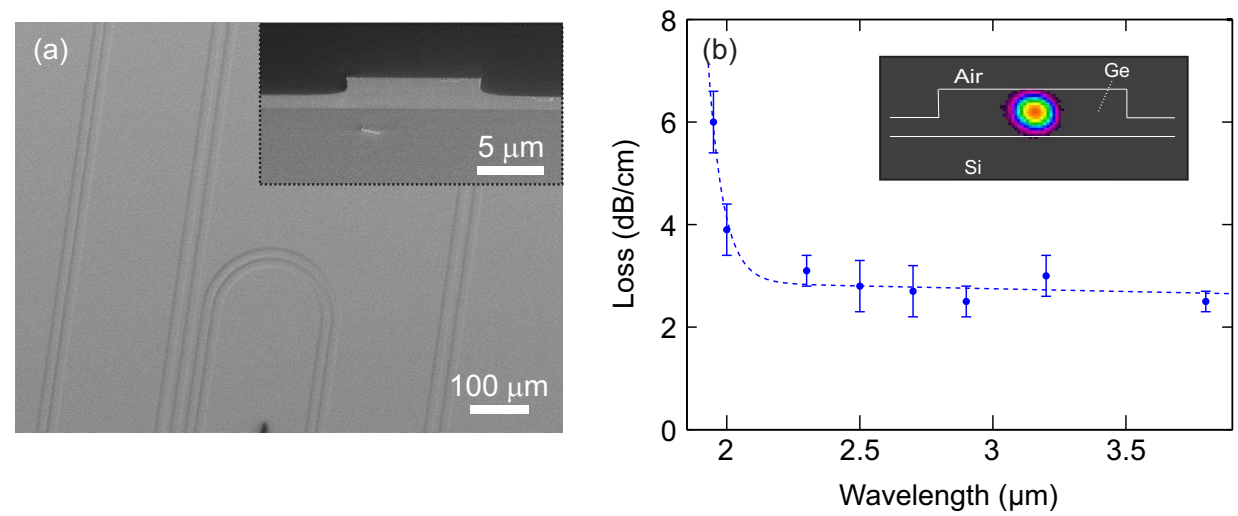

Fig. 1. (a) SEM micrograph of Ge-on-Si rib waveguides. (b) Linear losses over the $1.9-3.8 \mu \mathrm{m}$ wavelength range. Inset: mid-IR guided mode from the tapered output.

Rib waveguide structures were fabricated from Ge-on-Si wafers, with a $2 \mu \mathrm{m}$ thick germanium layer, using standard photolithography and chemical etching methods, as shown in Fig. 1(a) [3]. The waveguides used in this work were designed for operation across the $2-4 \mu \mathrm{m}$ wavelength region, with an etch depth of $1.2 \mu \mathrm{m}$ and a core width of $2.25 \mu \mathrm{m}$. To facilitate coupling, input and output tapers were fabricated at each end with a maximum width of $10 \mu \mathrm{m}$ (see inset in Fig. 1(a)). The propagation loss for the TE mode was measured using a cut-back method over the wavelength range $1.9-3.8 \mu \mathrm{m}$ using various mid-IR sources, with the results shown in Fig. 1(b). The output mode profile obtained at $2.5 \mu \mathrm{m}$ is displayed in the inset of Fig. 1(b), showing that it is well confined to the core. The high loss value recorded at $1.95 \mu \mathrm{m}$ is expected as it is close to germanium's band edge, however, beyond this the losses flatten out to a consistently low value of $\sim 3 \mathrm{~dB} / \mathrm{cm}$. 


\section{All-optical modulation}

To demonstrate all-optical modulation in the Ge-on-Si waveguides, a series of pump-probe experiments were performed. For the FCA-based modulation scheme, pump pulses operating at $1.5 \mu \mathrm{m}$ with a duration of $720 \mathrm{fs}$ were used to modulate a continuous wave (CW) source at selected wavelengths across $2-3.2 \mu \mathrm{m}$. The inset of Fig. 2(a) shows the modulation dynamics of a signal at $2 \mu \mathrm{m}$ recorded on a $10 \mathrm{GHz}$ InGaAS detector. By fitting the recovery with an exponential decay, the free-carrier lifetime can be estimated to be $\tau \sim 18 \mathrm{~ns}$. We note that as the lifetime determines the modulation rate, in this instance the operation speeds will be on the order of $\sim 50 \mathrm{MHz}$. Although it is not possible to directly measure the modulation depth, we can consider the pump induced carrier generation to be instantaneous when compared to the slow recovery time, which allow us to use the lifetime to place a lower bound on the extinction ratio of $4 \mathrm{~dB}$. Subsequent measurements revealed that the extinction ratio increases for increasing signal wavelength, with extinctions of $4.6 \mathrm{~dB}, 4.8 \mathrm{~dB}$, and $5.1 \mathrm{~dB}$ (as displayed in Fig. 2(a)) obtained for $2.6 \mu \mathrm{m}, 3 \mu \mathrm{m}$ and $3.2 \mu \mathrm{m}$, respectively.

In order to increase the modulation speed, our second setup looked to exploit the ultrafast TPA process via crossabsorption modulation (XAM). An amplified $5 \mathrm{ps}, 25 \mathrm{MHz}$ mode-locked fiber laser operating at $1.95 \mu \mathrm{m}$ was split into a high power pump and a weak probe pulse before they were overlapped within the Ge-on-Si waveguide as a function of delay. By using a chopper to modulate the probe signal, it is possible to map out the absorption due to the high power pump using a lock-in amplifier, as plotted in Fig.2(b). This curve clearly shows the ultrafast (ps) absorption dip induced on the probe that is generated via TPA, followed by a slower recovery due to slow free-carrier recombination. The extinction ratio is estimated to be around $8.1 \mathrm{~dB}$, higher than for the FCA modulation, and the ps response time indicates that modulation speeds of hundreds of $\mathrm{GHz}$ should be readily achievable. Finally, by comparing the XAM response with a fit obtained by solving the coupled nonlinear Schrödinger equations [4], it is possible to estimate the TPA parameter at the pump wavelength as $1280 \mathrm{~cm} / \mathrm{GW}$, in good agreement with the theoretical predictions [5].
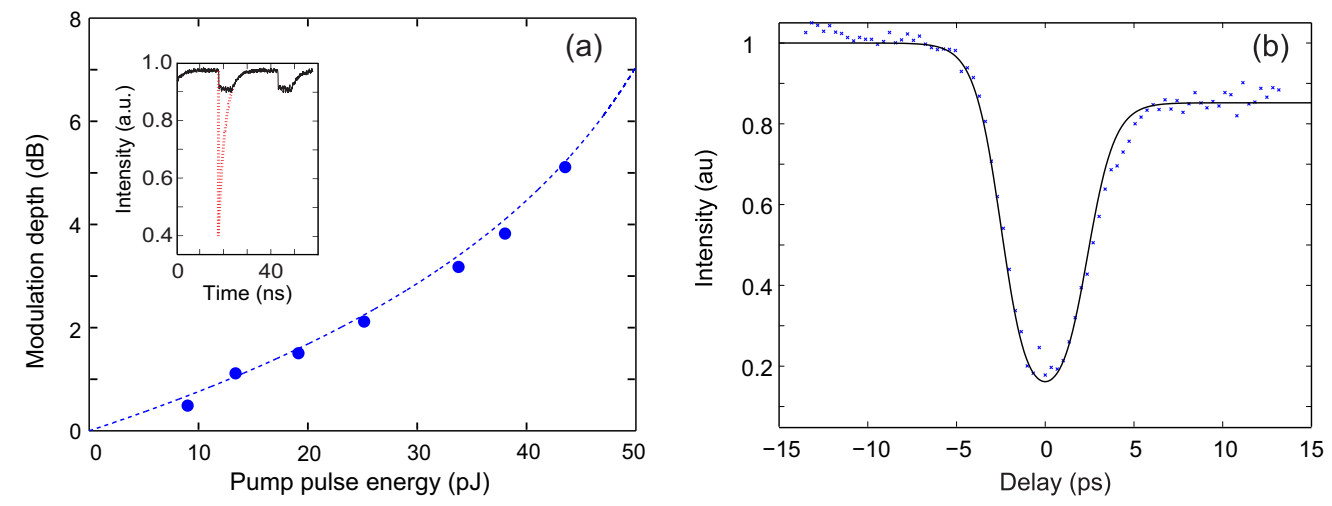

Fig. 2. (a) Measured modulation via FCA at $3.2 \mu \mathrm{m}$ (blue circles); inset shows the temporal dynamics of the modulation at $2 \mu \mathrm{m}$ together with an exponential fit (red curve). (b) Measured XAM at $1.95 \mu \mathrm{m}$ (blue dots) together with the simulated fit (black line).

\section{Conclusion}

We have characterized low loss Ge waveguides in the $2-3.8 \mu \mathrm{m}$ regime and demonstrated their use for all-optical modulation using both FCA and XAM schemes. We expect that all-optical modulation processes will play a key role in future mid-IR infrastructures, and these results highlight the suitability for Ge waveguides for use in this regime.

\section{References}

1. Y. C. Chang, V. Paeder, L. Hvozdara, J. M. Hartmann, and H. P. Herzig, "Low-loss germanium strip waveguides on silicon for the mid-infrared," Opt. Lett. 37, 2883-2885 (2012).

2. A. Malik, M. Muneeb, Y. Shimura, J. Van Campenhout, R.Loo, and G. Roelkens, "Germanium-on-Silicon Planar Concave Grating Wavelength (de)multiplexers in the Mid-Infrared," Appl. Phys. Lett. 103, 161119 (2013).

3. L. Shen, N. Healy, C. J. Mitchell, J. S. Penades, M. Nedeljkovic, G. Z. Mashanovich, and A. C. Peacock, "Mid-infrared all-optical modulation in low loss germanium-on-silicon waveguides," Opt. Lett. (2015) (In press).

4. P. Mehta, N. Healy, T. D. Day, J. R. Sparks, P. J. A. Sazio, J. V. Badding, and A. C. Peacock, "All-optical modulation using two-photon absorption in silicon core optical fibers," Opt. Express 19, 19078-19083 (2011).

5. N. K. Hon, R. Soref, and B. Jalali, "The third-order nonlinear optical coefficients of $\mathrm{Si}, \mathrm{Ge}$, and $\mathrm{Si}_{1-x} \mathrm{Ge}_{x}$ in the midwave and longwave infrared," App. Phys. Rev. 110, 011301 (2011). 